\title{
ChemComm
}

Check for updates

Cite this: Chem. Commun., 2018 54,5245

Received 23rd March 2018, Accepted 1st May 2018

DOI: $10.1039 / \mathrm{c} 8 \mathrm{cc} 03462 a$

rsc.li/chemcomm

\section{Dimensional crossover of correlated anion disorder in oxynitride perovskites $\$$}

\author{
Hannah Johnston, ${ }^{a}$ Ashley P. Black, ${ }^{b}$ Paula Kayser, (D) a Judith Oró-Solé, \\ David A. Keen, (D) ${ }^{c}$ Amparo Fuertes (D) ${ }^{b}$ and J. Paul Attfield (D) *a
}

\begin{abstract}
A simple crossover from two-dimensional to three-dimensional correlated disorder of $\mathrm{O}$ and $\mathrm{N}$ atoms on a cubic lattice has been discovered within the $\mathrm{Ba}_{1-x} \mathrm{Sr}_{x} \mathrm{TaO}_{2} \mathrm{~N}$ series of perovskite oxynitrides. The crossover is driven by lattice expansion as $x$ decreases, and provides a rapid increase in entropy due to a change from subextensive to extensive configurational entropy regimes.
\end{abstract}

Correlated disorders of atoms or magnetic moments, where local structure rules do not give rise to long range (crystallographic) order, have been identified in many crystalline materials such as water and spin ices, metal cyanides and molecular systems. ${ }^{1}$ Both two-dimensional (2D) and three-dimensional (3D) examples are known, but dimensional crossovers which are associated with changes of chemical or physical properties as new degrees of freedom become available are rare for correlated disorder. Dipolar spin-ice correlations in the pyrochlore $\mathrm{Dy}_{2} \mathrm{Ti}_{2} \mathrm{O}_{7}$ were switched from $3 \mathrm{D}$ to $2 \mathrm{D}$ behaviour through application of a magnetic field, ${ }^{2}$ but analogous atomic dimensional crossovers are not reported. Here we describe a simple strain-driven $2 \mathrm{D}$ to $3 \mathrm{D}$ crossover on a cubic lattice of correlated disorder of $\mathrm{O}$ and $\mathrm{N}$ atoms within the $\mathrm{Ba}_{1-x} \mathrm{Sr}_{x} \mathrm{TaO}_{2} \mathrm{~N}$ perovskites series.

$\mathrm{AMO}_{2} \mathrm{~N}$ or $\mathrm{AMON}_{2}$ oxynitride perovskites have useful properties such as water-splitting photocatalysis and high dielectric constants. $^{3,4} 2 \mathrm{D}$ correlations of $\mathrm{O} / \mathrm{N}$ atoms have been evidenced in many of these materials; ${ }^{5}$ from neutron and electron diffraction studies of $\mathrm{SrMO}_{2} \mathrm{~N}(\mathrm{M}=\mathrm{Nb}, \mathrm{Ta}){ }^{6} \mathrm{LaTaON}_{2}{ }^{7}$ and $\mathrm{RVO}_{2} \mathrm{~N}(\mathrm{R}=\mathrm{Pr}, \mathrm{Nd})^{8,9}$ perovskites, and from electron diffraction studies of $\mathrm{EuMO}_{2} \mathrm{~N}(\mathrm{M}=$ $\mathrm{Nb}, \mathrm{Ta})^{10}$ and $\mathrm{EuWON}_{2} \cdot{ }^{11}$ Anions within the $\mathrm{AMO}_{2} \mathrm{~N}$ perovskites are locally ordered as $\mathrm{MO}_{4} \mathrm{~N}_{2}$ octahedra where the two nitrides adopt a cis $\left(90^{\circ}\right)$ configuration which is favoured by the higher covalency of

\footnotetext{
${ }^{a}$ CSEC and School of Chemistry, University of Edinburgh, King's Buildings, Mayfield Road, Edinburgh, EH9 3JZ, UK. E-mail: j.p.attfield@ed.ac.uk

${ }^{b}$ Institut de Ciència de Materials de Barcelona (ICMAB-CSIC), Campus UAB, 08193 Bellaterra, Spain

${ }^{c}$ ISIS Facility, Rutherford Appleton Laboratory, Didcot, OX11 OQX, UK

$\dagger$ Open data for this paper are at https://datashare.is.ed.ac.uk/handle/10283/838. \$ Electronic supplementary information (ESI) available: $\mathrm{N}$ analysis, electron microscopy and further refinement results. See DOI: 10.1039/c8cc03462a
}

the M-N bond (Fig. 1a). ${ }^{12}$ Combining the cis configuration with the linear coordination of each nitride by two $\mathrm{M}$ cations results in the formation of zig zag $-\mathrm{M}-\mathrm{N}-$ chains and rings that spontaneously segregate into 2D planes within the perovskite structure, as shown in Fig. 1d. This is evidenced by a partial segregation of anions within the average unit cell (Fig. 1b) that is observable by neutron diffraction due to high $\mathrm{O} / \mathrm{N}$ scattering contrast. A chemical symmetry between the anion orders of $\mathrm{AMO}_{2} \mathrm{~N}$ and $\mathrm{AMON}_{2}$ perovskites obtains from interchanging $\mathrm{O}$ and $\mathrm{N}$ positions so that $2 \mathrm{D}$ layers of cis-TaO chains are found in $\mathrm{LaTaON}_{2}$. (a)

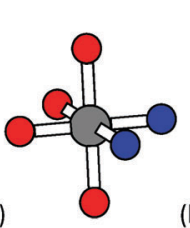

(b)

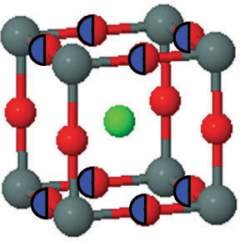

(c)

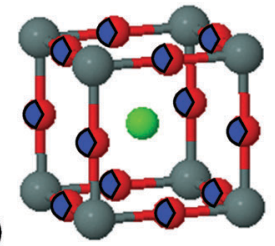

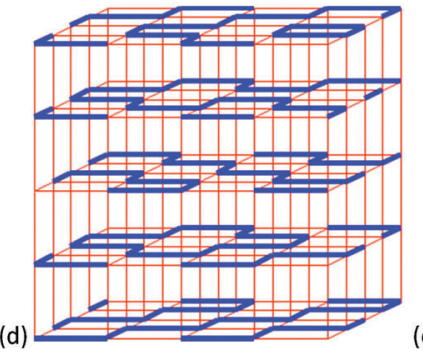

(e)

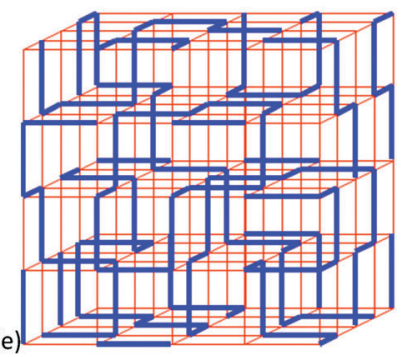

Fig. 1 Anion orders in $\mathrm{AMO}_{2} \mathrm{~N}$ perovskites, with $\mathrm{N} / \mathrm{O}$ atoms shown as blue/red throughout. (a) Local cis $\left(90^{\circ}\right)$ coordination of $\mathrm{N}$ atoms in all $\mathrm{MO}_{4} \mathrm{~N}_{2}$ octahedra. (b) The average anion distribution in 2D materials which have a tetragonal $P 4 / \mathrm{mmm}$ cell with two anion sites occupied $50 \%$ by $\mathrm{O}$ and $50 \%$ by $\mathrm{N}$, and one site of $100 \% \mathrm{O}$. (c) The average anion distribution in 3D materials which have the cubic $P m \overline{3} \mathrm{~m}$ perovskite structure with $67 \%$ occupancy of $\mathrm{O}$ and $33 \% \mathrm{~N}$ at all anion sites. (d) Local picture of cis-MN chains confined to 2D, where thick blue/thin red lines correspond to $\mathrm{M}-\mathrm{N}-\mathrm{M} / \mathrm{M}-\mathrm{O}-\mathrm{M}$ connections, and the average site occupancies correspond to those in (b). (e) Local picture of cis-MN chains propagating in 3D, giving the average site occupancies shown in (c). Correlated anion disorder in $\mathrm{AMON}_{2}$ perovskites is chemically symmetric through reversal of $O$ and $N$ in all figures. 
An alternative 3D distribution of the cis-chains in oxynitride perovskites is also possible (Fig. 1e), but no evidence for a 2D to 3D crossover was found in high temperature experiments. Chains were found to be confined to $2 \mathrm{D}$ up to the highest measured temperature of $1100{ }^{\circ} \mathrm{C}$ in neutron diffraction studies of $\mathrm{SrTaO}_{2} \mathrm{~N}$ and $\mathrm{LaTaON}_{2} \cdot{ }^{7}$ However, while a neutron diffraction and PDF (pair distribution function) study of $\mathrm{BaTaO}_{2} \mathrm{~N}$ reported that cis- $\mathrm{TaO}_{4} \mathrm{~N}_{2}$ octahedra were present, it was not clear whether $\mathrm{N}$ atoms are distributed in $2 \mathrm{D}$ or $3 \mathrm{D} .{ }^{13}$ We have therefore explored the full series of solid solutions $\mathrm{Ba}_{1-x} \mathrm{Sr}_{x} \mathrm{TaO}_{2} \mathrm{~N}(0 \leq x \leq 1)$ using high resolution neutron and X-ray diffraction to establish the $2 \mathrm{D} / 3 \mathrm{D}$ nature and evolution of the correlated anion chain disorder.

Highly crystalline powder samples of the solid solutions $\mathrm{Ba}_{1-x} \mathrm{Sr}_{x} \mathrm{TaO}_{2} \mathrm{~N}(x=0,0.2,0.4,0.6,0.8$ and 1$)$ were prepared using stoichiometric amounts of $\mathrm{BaCO}_{3}$ (Alfa Aesar 99.997\%), $\mathrm{SrCO}_{3}$ (Alfa Aesar 99.994\%) and $\mathrm{Ta}_{3} \mathrm{~N}_{5}$ (made by ammonolysis of $\mathrm{Ta}_{2} \mathrm{O}_{5}$ (Aldrich $99.99 \%$ ) at $850{ }^{\circ} \mathrm{C}$ ). A pellet was fired at $950{ }^{\circ} \mathrm{C}$ for $6 \mathrm{~h}$ and then $1500{ }^{\circ} \mathrm{C}$ for $3 \mathrm{~h}$ under a $5 \% \mathrm{H}_{2} / 95 \% \mathrm{~N}_{2}$ gas mixture (Air Liquide). The sample was placed in a molybdenum crucible and covered with a zirconium foil to scavenge oxygen and water. Nitrogen contents from chemical analysis were found to be between 0.95(2) and 0.99(2) $\mathrm{N}$ atoms per formula unit without any systematic variation with Sr content $x$ showing that the materials are essentially stoichiometric.

High resolution X-ray and neutron diffraction profiles were recorded for all of the samples at $300 \mathrm{~K}$. Synchrotron X-ray powder diffraction data were measured from capillary samples at the MSPD beamline ${ }^{14}$ of the ALBA Synchrotron (Cerdanyola del Vallès, Spain). Radiation with wavelength $\lambda=0.6263 \AA$ was selected by a double $\mathrm{Si}(111)$ crystal monochromator and calibrated with NIST Si powder. Neutron powder diffraction data were collected on the High Resolution Powder Diffractometer (HRPD) at the ISIS spallation neutron source, Rutherford Appleton Laboratory, UK. Approximately $1 \mathrm{~g}$ of each sample was used in a vanadium can and diffraction patterns were recorded for 2 hours. Neutron and X-ray powder data were analysed separately by Rietveld refinement using the Fullprof program. ${ }^{15}$ In the neutron analysis, structural models were refined against data collected from the backscattering bank which provides a $d$ range of 0.65-2.5.

Previous neutron studies have shown that $\mathrm{SrTaO}_{2} \mathrm{~N}$ has a superstructure at $300 \mathrm{~K}$ due to ordered rotations of the $\mathrm{TaO}_{4} \mathrm{~N}_{2}$ octahedra. ${ }^{6,7}$ However, the $x=1$ sample used in this investigation was found to have very broad superstructure peaks indicating that the rotational domains are small, and this is confirmed by electron diffraction patterns of individual microcrystallites. Further details are in ESI. $\neq$ Complete suppression of the superstructure was recently reported in another study where $\mathrm{SrTaO}_{2} \mathrm{~N}$ was also prepared by high temperature nitrogen treatment. ${ }^{16}$ No rotational superstructure peaks were observed in the powder neutron diffraction profiles of the other $\mathrm{Ba}_{1-x} \mathrm{Sr}_{x} \mathrm{TaO}_{2} \mathrm{~N}$ samples. Hence fits of the simple tetragonal P4/mmm (Fig. 1b) or cubic Pm $\overline{3} m$ (Fig. 1c) models were used to determine the degree of anion order for all samples.

The high $\Delta d / d$ resolutions of the HRPD and MSPD instruments enable the $P 4 / \mathrm{mmm}$ model to be tested, although the tetragonal lattice distortion is extremely small and all $\mathrm{Ba}_{1-x} \mathrm{Sr}_{x} \mathrm{TaO}_{2} \mathrm{~N}$ patterns appear cubic by eye, as shown in Fig. 2 and ESI. $\ddagger$ For

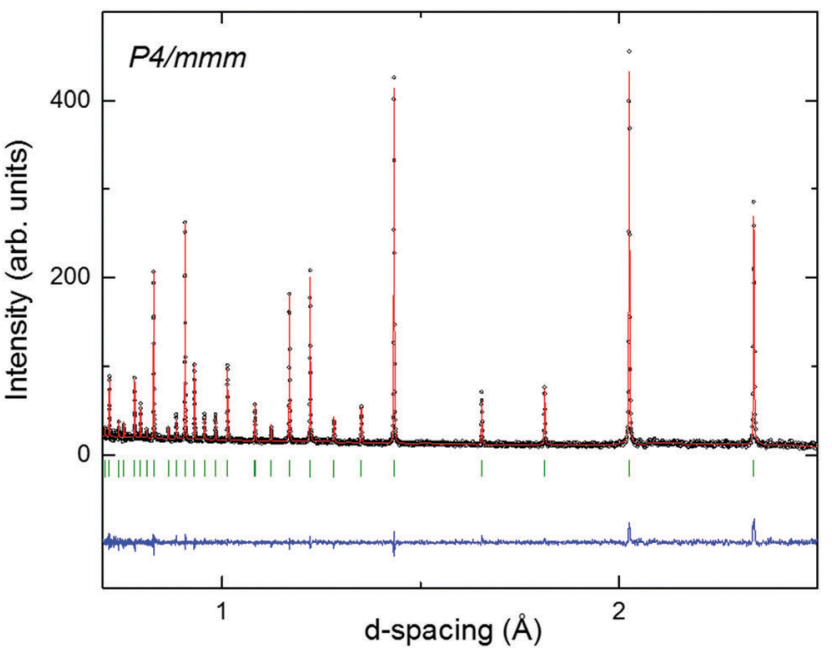

Fig. 2 Fit of the tetragonal P4/mmm model in Fig. 1(b) describing $2 \mathrm{D}$-chains to room temperature neutron powder diffraction data for $\mathrm{Ba}_{0.2} \mathrm{Sr}_{0.8} \mathrm{TaO}_{2} \mathrm{~N}$. Residuals; $\chi^{2}=1.17, R_{\mathrm{wp}}=8.03 \%$.

$x=0.4-1.0$ samples, the tetragonal $P 4 / \mathrm{mmm}$ model was found to give stable neutron refinements with consistent tetragonal strains $\left((a-c) / a \approx 10^{-4}\right)$, as shown in Fig. 3, and refined $\mathrm{O} / \mathrm{N}$ site occupancies within $\pm 7 \%$ of the ideal values for the $2 \mathrm{D}$ cis-chains model, as in Fig. 4. Hence the 2D correlated disorder previously found for $x=1 \mathrm{SrTaO}_{2} \mathrm{~N}$ is demonstrated to extend across the $x=0.4-1.0$ range. In contrast, neutron refinements of tetragonal lattice parameters and $\mathrm{O} / \mathrm{N}$ site occupancies were unstable for the $x=0$ and 0.2 samples, and so these data were fitted with the cubic $P m \overline{3} m$ model that is consistent with 3D correlated disorder.

The X-ray data do not provide $\mathrm{O} / \mathrm{N}$ scattering contrast but do enable the tetragonal lattice distortion to be refined. Stable refinements of a $P 4 / \mathrm{mmm}$ tetragonal perovskite cell were obtained for $x=0.4-1.0$ samples, but the refinements were unstable for fits to $x=0$ and $x=0.2$ data, corroborating the neutron results that these two samples have true cubic $P m \overline{3} m$ lattice symmetry. Hence both high resolution powder neutron and X-ray results demonstrate that a subtle change from cubic $P m \overline{3} \mathrm{~m}$ to tetragonal $P 4 / \mathrm{mmm}$ symmetry occurs between $x=0.2$ and 0.4 , with similar variations in the tetragonal lattice distortion (Fig. 3), and consistent with the neutron occupancies in Fig. 4. The variations of these quantities do not correlate with the refined temperature factors shown in ESI.

The cubic $\mathrm{Ba}_{1-x} \mathrm{Sr}_{x} \mathrm{TaO}_{2} \mathrm{~N}$ structure at $x=0-0.2$ is consistent with a $3 \mathrm{D}$ distribution of disordered cis-chains (Fig. 1e), or at least small local domains of $2 \mathrm{D}$ propagation distributed over the three possible orientations, while neutron occupancies show that long range $2 \mathrm{D}$ confinement of anion chain layers is present across the tetragonal $x=0.4-1$ samples (Fig. 1d). The absence of an intermediate $P 4 / \mathrm{mmm}$ structure with $\mathrm{O} / \mathrm{N}$ occupancies between the $2 \mathrm{D}$ and $3 \mathrm{D}$ distributions (i.e. oxygen occupancy $\approx 70-90 \%$ in Fig. 4) indicates that the crossover is relatively sharp with different thermodynamic factors favouring $2 \mathrm{D}$ or $3 \mathrm{D}$ correlations.

Although both the $2 \mathrm{D}$ and $3 \mathrm{D}$ correlated arrangements (Fig. 1d and e) appear disordered by eye, previous theoretical 


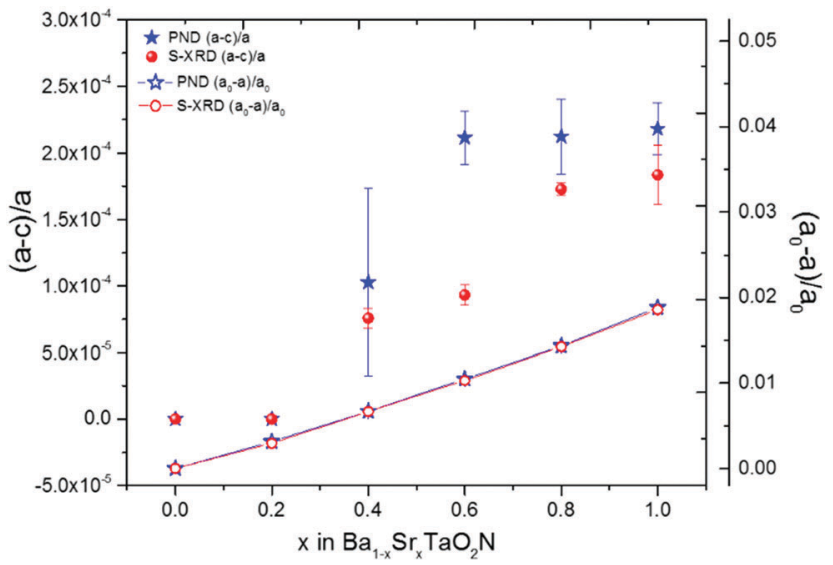

Fig. 3 Variations of the tetragonal strain $(a-c) / a$ (left scale, filled points) and compressive cubic strain $\left(a_{0}-a\right) / a_{0}$ (right scale, open points; $a_{0}$ is the lattice parameter at $x=0$ ) across the $\mathrm{Ba}_{1-x} \mathrm{Sr}_{x} \mathrm{TaO}_{2} \mathrm{~N}$ series with the $x=0-0.2$ data from cubic perovskite structure $P m \overline{3} m$ refinements and the $x=0.4-1$ data from tetragonal perovskite structure $P 4 / \mathrm{mmm}$ refinements. Red/blue points are from neutron/X-ray refinements.

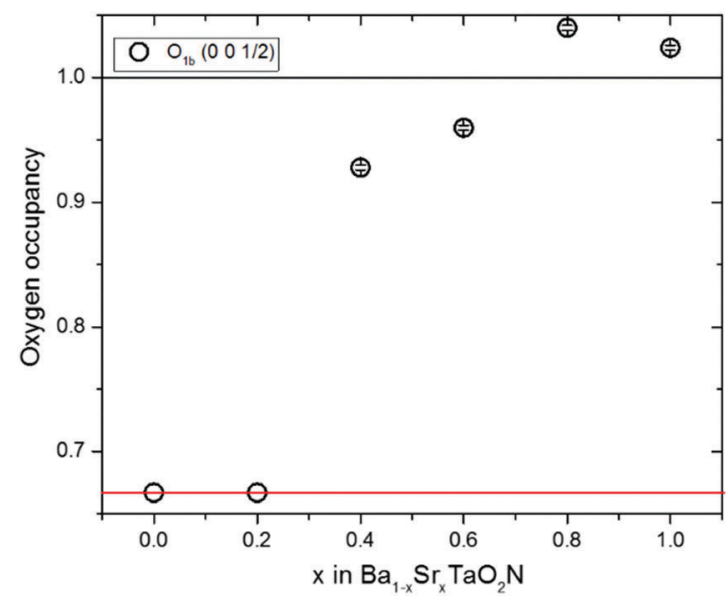

Fig. 4 Plot of oxygen occupancies for the $c$-axis anion site from neutron refinements against $x$ in the $\mathrm{Ba}_{1-x} \mathrm{Sr}_{x} \mathrm{TaO}_{2} \mathrm{~N}$ series. Values for the $x=0-0.2$ cubic $P m \overline{3} m$ refinements are fixed at 0.67 corresponding to the model in Fig. $1 \mathrm{c}$ which averages over the 3D cis-TaN chains in Fig. 1e. Occupancies for the $x=0.4-1$ samples are refined against the tetragonal $\mathrm{P} 4 / \mathrm{mmm}$ model and approximate to those shown in Fig. $1 \mathrm{~b}$ which averages the 2D cis-TaN chains model in Fig. $1 d$

analysis has shown that their configurational entropies are very different. ${ }^{17}$ The $2 \mathrm{D}$ arrangement found in $x=1 \mathrm{SrTaO}_{2} \mathrm{~N}$ has a subextensive molar entropy that varies with the number of atoms $N$ per particle as $S=(2 R \ln 2) / N^{1 / 3}$. The highly crystalline $\mathrm{Ba}_{1-x} \mathrm{Sr}_{x} \mathrm{TaO}_{2} \mathrm{~N}$ powders used here have particle sizes $\sim 1 \mu \mathrm{m}$ with $N \approx 1.6 \times 10^{10}$ so $S \approx 6 \times 10^{-4} R$ and hence configurational entropy is very small for the $2 \mathrm{D}$ materials. This unusual structural state has been described as an 'open order' based on the closure properties of the set of vectors for long range structural correlation. ${ }^{17}$ The $3 \mathrm{D}$ arrangement of chains found in $x=0 \mathrm{BaTaO}_{2} \mathrm{~N}$ has a conventional configurational entropy ('closed order') that scales extensively, with value $S=2 R \ln (4 / 3) \approx$ $0.6 R$. Hence the $2 \mathrm{D}$ to $3 \mathrm{D}$ crossover in anion chain correlation behaves thermodynamically as an order to disorder transition with an increase of $0.6 R \approx 4.8 \mathrm{~J} \mathrm{~K}^{-1} \mathrm{~mol}^{-1}$ in configurational entropy. This must be balanced by some $\Delta H=T \Delta S$ enthalpy stabilisation of the $2 \mathrm{D}$ state at the crossover, and as the present samples were equilibrated at $1500{ }^{\circ} \mathrm{C}$ the stabilisation of the $2 \mathrm{D}$ state relative to the $3 \mathrm{D}$ is estimated as $\Delta H \approx 8.5 \mathrm{~kJ} \mathrm{~mol}^{-1}$. This stabilisation most likely derives from conjugation energy when all $\mathrm{Ta}-\mathrm{N}$ bonds are co-planar, analogous to the stabilisation of planar structures for aromatic organic molecules, and so is very sensitive to increasing interatomic separation which lowers Ta-N orbital hybridisation. Hence the lattice expansion as $x$ decreases (shown as a decreasing compressive cubic lattice strain on the right hand scale in Fig. 3) drives the $2 \mathrm{D}$ to $3 \mathrm{D}$ crossover in the $\mathrm{Ba}_{1-x} \mathrm{Sr}_{x} \mathrm{TaO}_{2} \mathrm{~N}$ series.

In conclusion, this study demonstrates that a sharp dimensional crossover of correlated atomic disorder occurs near $x=0.2$ in the $\mathrm{Ba}_{1-x} \mathrm{Sr}_{x} \mathrm{TaO}_{2} \mathrm{~N}$ series. The $2 \mathrm{D}$ phase is destabilised by lattice expansion as $x$ decreases, and the release of structural degrees of freedom provides a rapid increase in entropy due to a change from subextensive to extensive configurational entropy regimes.

We thank STFC, UK for support for H. J. and provision of ISIS beamtime, and EPSRC for additional support. This work was also supported by the Ministerio de Economia y Competitividad (MINECO), Spain through Project MAT2017-86616-R, the Severo Ochoa Program SEV-2015-0496, and Fellowship support to AB (MAT2011-24757). We thank the ALBA synchrotron for the provision of beamtime and Prof. Rosa Palacin, Dr Carlos Frontera (ICMAB-CSIC) and Dr F. Fauth (ALBA) for assistance with data collection.

\section{Conflicts of interest}

There are no conflicts to declare.

\section{References}

1 D. A. Keen and A. L. Goodwin, Nature, 2015, 521, 303.

2 Y. Tabata, H. Kadowaki, K. Matsuhira, Z. Hiroi, N. Aso, E. Ressouche and B. Fåk, Phys. Rev. Lett., 2006, 97, 257205.

3 A. Fuertes, Mater. Horiz., 2015, 2, 453.

4 A. Fuertes, J. Mater. Chem., 2012, 22, 3293.

5 J. P. Attfield, Cryst. Growth Des., 2013, 13, 4623.

6 M. Yang, J. Oró-Solé, J. A. Rodgers, A. B. Jorge, A. Fuertes and J. P. Attfield, Nat. Chem., 2011, 3, 47.

7 L. Clark, J. Oró-Solé, K. S. Knight, A. Fuertes and J. P. Attfield, Chem. Mater., 2013, 25, 5004.

8 J. Oró-Solé, L. Clark, W. Bonin, J. P. Attfield and A. Fuertes, Chem. Commun., 2013, 49, 2430.

9 L. Clark, N. Kumar, W. Bonin, A. Sundaresan, J. P. Attfield, C. N. R. Rao and A. Fuertes, J. Mater. Chem. C, 2014, 2, 2212.

10 A. B. Jorge, J. Oro-Sole, A. M. Bea, N. Mufti, T. T. M. Palstra, J. A. Rodgers, J. P. Attfield and A. Fuertes, J. Am. Chem. Soc., 2008, 130, 12572.

11 M. Yang, J. Oro-Sole, A. Kusmartseva, A. Fuertes and J. P. Attfield, J. Am. Chem. Soc., 2010, 132, 4822.

12 H. Wolff and R. Dronskowski, J. Comput. Chem., 2008, 29, 2260.

13 K. Page, M. W. Stoltzfus, Y.-I. Kim, T. Proffen, P. M. Woodward, A. K. Cheetham and R. Seshadri, Chem. Mater., 2007, 19, 4037.

14 F. Fauth, I. Peral, C. Popescu and M. Knapp, Powder Diffr., 2013, 28, S360.

15 J. Rodríguez-Carvajal, Commission on Powder Diffraction (IUCr) Newsletter, 2001, 26, 12.

16 D. Chen, D. Habu, Y. Masubuchi, S. Torii, T. Kamiyama and S. Kikkawa, Solid State Sci., 2016, 54, 2.

17 P. J. Camp, A. Fuertes and J. P. Attfield, J. Am. Chem. Soc., 2012, 134, 6762. 